\title{
MicroRNA-93-5p may participate in the formation of morphine tolerance in bone cancer pain mouse model by targeting Smad5
}

\author{
Wen-Feng Xiao, ${ }^{1, *}$, Yu-Sheng $\mathrm{Li}^{1,}{ }^{,}$, Wei Lou ${ }^{2}$, Ting Cai ${ }^{3}$, Shun Zhang ${ }^{4}$, Xiao-Ying $\mathrm{Hu}^{5}$, \\ Xing-Wang Zhang ${ }^{6}$, Wei Luo ${ }^{1}$ \\ ${ }^{1}$ Department of Orthopaedics, Xiangya Hospital, Central South University, Changsha 410008, China \\ ${ }^{2}$ Department of Pain Treatment, Ningbo No.2 Hospital, Ningbo 315010, China \\ $3^{3}$ Department of Emergency Internal Medicine, Ningbo No.2 Hospital, Ningbo 315010, China \\ ${ }^{4}$ Stem Cell Laboratory, Ningbo No.2 Hospital, Ningbo 315010, China \\ ${ }^{5}$ Department of Nephropathy Dialysis Center, Shuguang Hospital Affiliated to Shanghai University of Traditional Chinese \\ Medicine, Shanghai 201203, China \\ ${ }^{6}$ Division of Pharmaceutics, College of Pharmacy, Jinan Unversity, Guangzhou 510632, P.R. China \\ *These authors have contributed equally to this work \\ Correspondence to: Wei Luo, email: lvowei0928@126.com \\ Ting Cai, email: Addison_7172@126.com \\ Keywords: microRNA-93-5p, Smad5, bone cancer pain, morphine tolerance, paw mechanical withdrawal threshold \\ Received: February 29, $2016 \quad$ Accepted: June 30,2016 Published: July 11, 2016
}

\section{ABSTRACT}

Objective: In this study, we aim to find out the role of microRNA-93-5p (miR-93) and Smad5 in morphine tolerance in mouse models of bone cancer pain (BCP).

Results: At 7 days after injection of morphine, the PMWT showed no significant difference between the morphine model group and the saline model group $(P<$ 0.05 ), suggesting that morphine tolerance had formed in the morphine model group. The morphine model group had higher miR-93 expression and lower Smad5 mRNA expression than the saline model group. Smad5 is a downstream target gene of miR93. At 7, 9 and 14 days after injection of lentiviruses, the L/anti-miR-93 group had the lowest PMWTs, while the Smad5 shRNA group presented the highest PMWTs among these five groups (all $P<0.05$ ).

Methods: We built mouse models of BCP and morphine tolerance and recorded $50 \%$ PMWT. After 6 days of modeling, we set saline control group, morphine control, saline model group and morphine model group (morphine tolerance emerged). We performed luciferase reporter gene assay to verify the relation between miR-93 and Smad5. After lentivirus transfection, the mice with morphine tolerance were assigned into L/anti-miR-93 group, Smad5 shRNA group, L/anti-miR-93 + Smad5 shRNA group, blank group and PBS control group. RT-qPCR, Western Blot assay and immumohistochemical staining were performed to observe the changes of miR-93 and Smad5.

Conclusion: Up-regulation of miR-93 may contribute to the progression of morphine tolerance by targeting Smad5 in mouse model of BCP.

\section{INTRODUCTION}

Bone is the most frequently site to be affected through cancer metastasis which has been implicated in lung cancer, breast cancer, as well as hepatocellular carcinoma due to the prevalence of these diseases. At postmortem examination, up to $70 \%$ of patients dying of these cancers presents bone metastatic [1-3]. Also, in the United States, it has been estimated that tumor metastasis to the bone affects more than 400,000 persons each year [2]. This bone metastasis included a cycle of tumor growth, bone destruction, and formation of woven 
Table 1: Recordings of the PMWT of all groups during the establishment of BCP model and morphine tolerance model

\begin{tabular}{|c|c|c|c|c|c|c|c|c|}
\hline & \multirow{2}{*}{$\begin{array}{l}\text { Pre-surgery } \\
\text { (g) }\end{array}$} & \multicolumn{3}{|c|}{ Post-surgery (g) } & \multicolumn{4}{|c|}{ After morphine injection (g) } \\
\hline & & 3d & 6d & 9d & 1d & $3 d$ & $5 d$ & $7 d$ \\
\hline $\begin{array}{l}\text { Saline } \\
\text { control } \\
\text { group }\end{array}$ & $6.35 \pm 0.57$ & $6.42 \pm 0.69$ & $6.41 \pm 0.74$ & $6.56 \pm 0.63$ & $6.51 \pm 0.77$ & $6.62 \pm 0.79$ & $6.61 \pm 0.84$ & $6.57 \pm 0.73$ \\
\hline $\begin{array}{l}\text { Morphine } \\
\text { control } \\
\text { group }\end{array}$ & $6.38 \pm 0.78$ & $6.44 \pm 0.74$ & $6.39 \pm 0.60$ & $6.45 \pm 0.73$ & $8.48 \pm 0.97^{*}$ & $8.64 \pm 0.83^{*}$ & $8.03 \pm 0.74^{*}$ & $6.65 \pm 0.66$ \\
\hline $\begin{array}{l}\text { Saline } \\
\text { model } \\
\text { group }\end{array}$ & $6.32 \pm 0.65$ & $6.08 \pm 0.53$ & $5.53 \pm 0.56^{* \&}$ & $3.65 \pm 0.32^{* \&}$ & $3.51 \pm 0.37$ & $3.67 \pm 0.36$ & $3.83 \pm 0.39$ & $4.32 \pm 0.43$ \\
\hline $\begin{array}{l}\text { Morphine } \\
\text { model } \\
\text { group }\end{array}$ & $6.30 \pm 0.20$ & $6.01 \pm 0.58$ & $5.34 \pm 0.49^{\Delta \&}$ & $3.60 \pm 0.35^{\Delta \&}$ & $7.58 \pm 0.75^{\#}$ & $7.98 \pm 0.84^{\#}$ & $5.88 \pm 0.61^{\#}$ & $4.40 \pm 0.46$ \\
\hline
\end{tabular}

PMWT, paw mechanical withdrawal threshold; BCP, bone cancer pain; ${ }^{*}, P<0.05$ in comparison with the saline control group; ${ }^{\Delta}, P<0.05$ in comparison with the morphine control group; ${ }^{*}, P<0.05$ in comparison with the saline model group; $\&, P<0.05$ in comparison with pre-treatment.

bone begins, inevitably bringing about considerable pain, that is, bone cancer pain (BCP) [4]. Bone cancer pain is prevalent among cancer patients and can have a devastating effect on their quality of life [5]. Morphine is a conventionally-selected pure $\mu$-agonist opioid for pain relief in cancer patients [6]. Unfortunately, prolonged takein of morphine may lead to cellar or molecular adaptions and therewith produce morphine tolerance $[7,8]$. In this context, for more satisfactory pain relief in cancer patients, more efforts should be made to figure out the mechanism of morphine tolerance at the molecular level.

MicroRNA-93-5p (miR-93) has also been suggested as a contributing factors in disease progression trough regulation of it downstream targets, including P21, cyclin B1, ERBB2, Akt3, SOX4, PTEN, STAT3, vascular endothelial growth factor A and Smad7 [9-13]. In lung cancer, miR-93 may target LATS2 to enhance metastasis or downregulate NEDD4L to promote TGF- $\beta$-induced epithelial-to-mesenchymal transition, an important mechanism for cancer metastasis $[14,15]$. Using certain biology software, we find that Smad5 is also a target gene of miR-93. As suggested, bone morphogenetic protein (BMP)/Smad signaling pathway promote the vascular development [16]. As a key mediator of BMP/ Smad pathway, Smad5 can act as a target of microRNA to suppress the endothelial development and thereby the angiogenesis, a must for tumor progression and tumor metastases [17, 18]. Interestingly, Smad5 is a wellaccepted suppressive gene in osteoclast differentiation and thereby decreases resorption pits [19]. To our knowledge, through these resorption pits, osteoclasts resorb bone, stimulating nerve fiber-rich TRPV1 or ASIC3 channels and consequently result in $\mathrm{BCP}$ [4]. On basis of all the facts, we speculate that miR-93 and Smad5 may correlate with the formation and progression of morphine tolerance.

In the present study, we build a BCP model with Lewis lung cancer cells, aiming to investigate the association between miR-93 and Smad5 and the effect of miR-93 and Smad5 on morphine tolerance.

\section{RESULTS}

\section{PMWT in the BCP model and the morphine tolerance model}

Table 1 showed the PMWTs before modeling and at $3^{\text {rd }}, 6^{\text {th }}$ and $9^{\text {th }}$ day after modeling and at $1^{\text {st }}, 3^{\text {rd }}, 5^{\text {th }}$ and $7^{\text {th }}$ day after injection of morphine. As presented, at $6^{\text {th }}$ and $9^{\text {th }}$ day after modeling, the PMWT was significantly higher in the saline model group and in the morphine model group than that in the saline control group and in the morphine control group, correspondingly (the saline model vs. the saline control, $P<0.05$; the morphine model vs. the morphine control, $P<0.05$ ); the PMWTs in both saline model and morphine model groups were significantly lower at 6 days after treatment in comparison with pretreatment (both $P<0.05$ ). The above results suggested that BCP model was successfully built. At $7^{\text {th }}$ day after injection of morphine, the PMWT showed no significant difference between the morphine model group and the 
saline model group $(P<0.05)$, suggesting that morphine tolerance had formed in the morphine model group.

\section{miR-93-5p expression and Smad5 mRNA and protein expression after morphine tolerance}

Our RT-qPCR demonstrated that, the saline model group had a higher miR-93-5p expression and lower
Smad5 mRNA expression than the saline control group, but had a lower miR-93-5p expression and a higher Smad5 mRNA expression than the morphine model group (all $P<0.05$ ) (Figure 1A, 1B). The above results indicated that the miR-93-5p expression was in negative association with the Smad5 mRNA expression $(P<0.05)$ (Figure 1C). Besides, we detected the Smad5 expression in the four group by Western Blot Assay (Figure 1D)

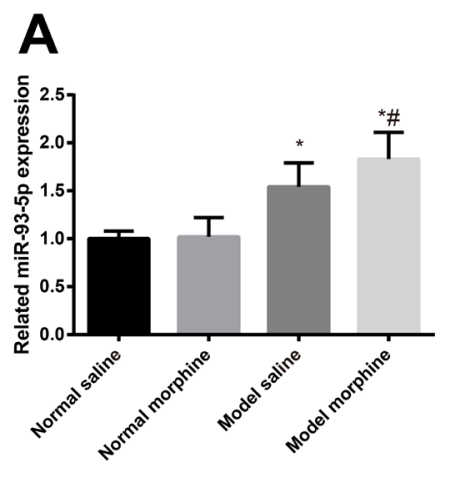

D

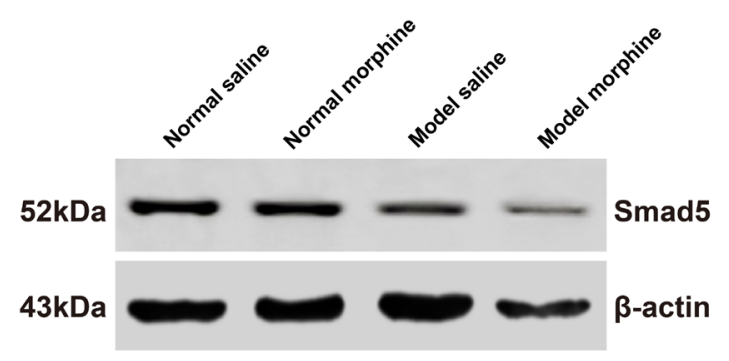

$\mathbf{F}$

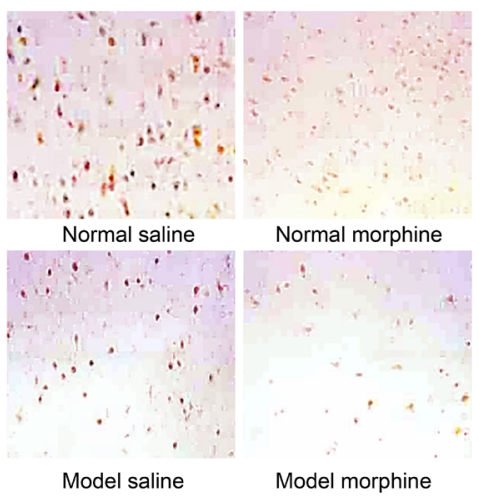

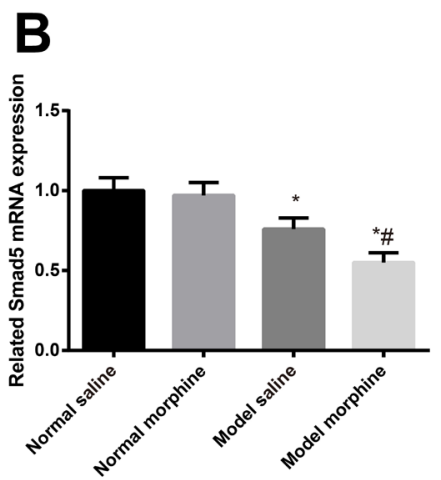
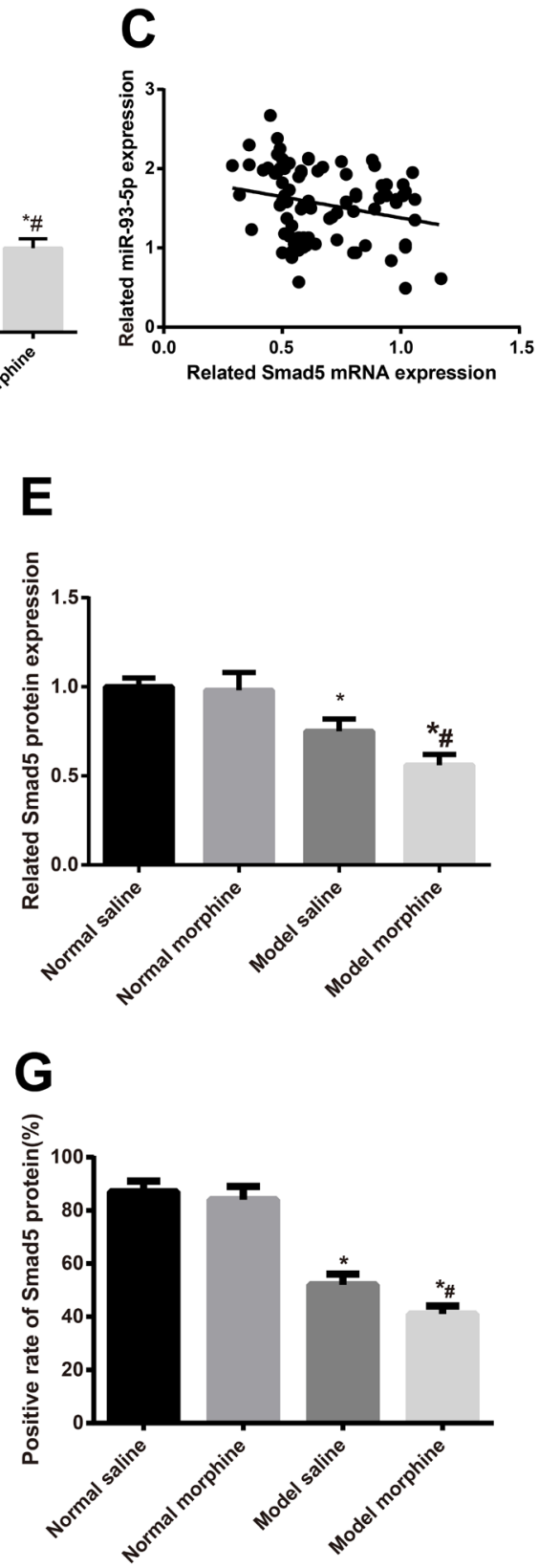

Figure 1: After the morphine tolerance model was built, miR-93-5p expression and Smad5 mRNA and protein expressions were detected. A. miR-93-5p expression; B. Smad5 mRNA expression; C. miR-93-5p expression was in negative correlation with Smad5 mRNA expression; D. electrophoretogram of Smad5 protein by Western blot; E. alternations of Smad5 protein expression; F. Smad5 protein expression in L5 spinal marrows by immunohistochemistry; G. semi-quantitative Smad5 protein expression in L5 spinal marrows by immunohistochemistry.

Note: ${ }^{*}, P<0.05$ in comparison with the saline control group; $\#, P<0.05$ in comparison with the saline model group. 
and immunohistochemical staining (Figure 1E), finding that, among the four group, the saline model group had a lower Smad5 expression than the saline control group but a higher Smad5 expression than the morphine model group(both $P<0.05$ ).

\section{miR-93 directly targets Smad5}

We synthesized a sequence (GCACTTT) at target site (WT) and then a mutant sequence Mut (CGTGAAA) through site-directed mutagenesis in WT (Figure 2A) to conduct a dual luciferase reporter gene assay. The result of the luciferase reporter gene assay showed that the fluorescence intensity showed no significant difference between Group C and Group D while exhibited obvious difference between Group A and Group B $(P<0.05)$ (Figure 2B). This result suggested thatSmad5 was a target gene of miR-93-5p.

\section{PMWT in the mice with morphine tolerance after transfection with lentiviruses}

As shown in Table 2, the L/anti-miR-93 group had the lowest PMWTs, while the Smad5 shRNA group presented the highest PMWTs among these five groups (all $P<0.05)$. No such difference was identified among the L/ anti-miR-93-5p + Smad5 shRNA group, the blank group and the PBS control group (all $P>0.05$ ). After the last measurement of PMWT at $14^{\text {th }}$ day after injection, the L5 spinal marrow in the mice in the five groups were selected for the observation of virus transfection. As shown in Figure 3, the L/anti-miR-93-5p group, the Smad5 shRNA group and the L/anti-miR-93-5p + Smad5 shRNA group, three of them had a transfection efficiency of about $80 \%$. Meanwhile, using the slices, we detected also miR-93$5 \mathrm{p}$ expression and Smad5 mRNA expression with RTqPCR (Figure 4A-4B) and protein expressionSmad5 with Western Blot Assay and immunohistochemical staining (Figure 4C-4F). We found that the miR-93-5p expression was significantly lower in the L/anti-miR-93-5p group and the L/anti-miR-93-5p + Smad5 shRNA group than that in the other three groups (all $P<0.05$ ); however, it presented no difference among the other three groups (all $P>0.05)$. As revealed, both the Smad5 mRNA and protein expressions had the highest value in the L/anti-miR-935 p group while the lowest value in the Smad5 shRNA group among the five groups (all $P<0.05$ ), and no such difference was observed among the L/anti-miR-93-5p + Smad5 shRNA group, the blank group and the PBS control group (all $P>0.05$ ).

\section{DISCUSSION}

Usually, the analgesic effect of morphine is realized by upregulation of pain threshold, and patients treated with morphine could endure more intense pain [25]. However, the painkiller effect is decreased along with lasting administration of morphine at a constant dose [26,
A

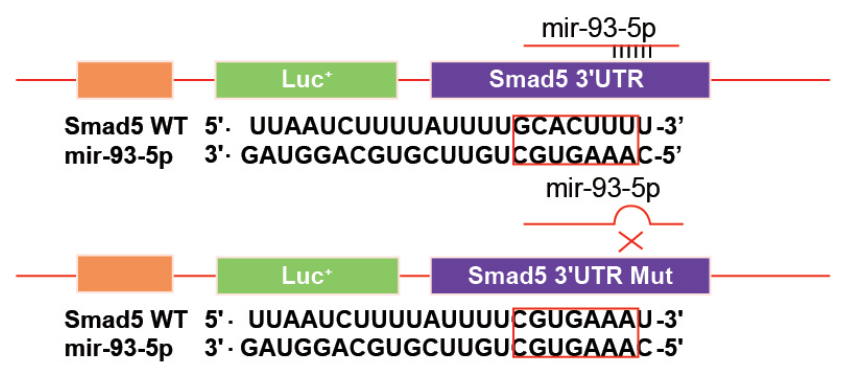

B

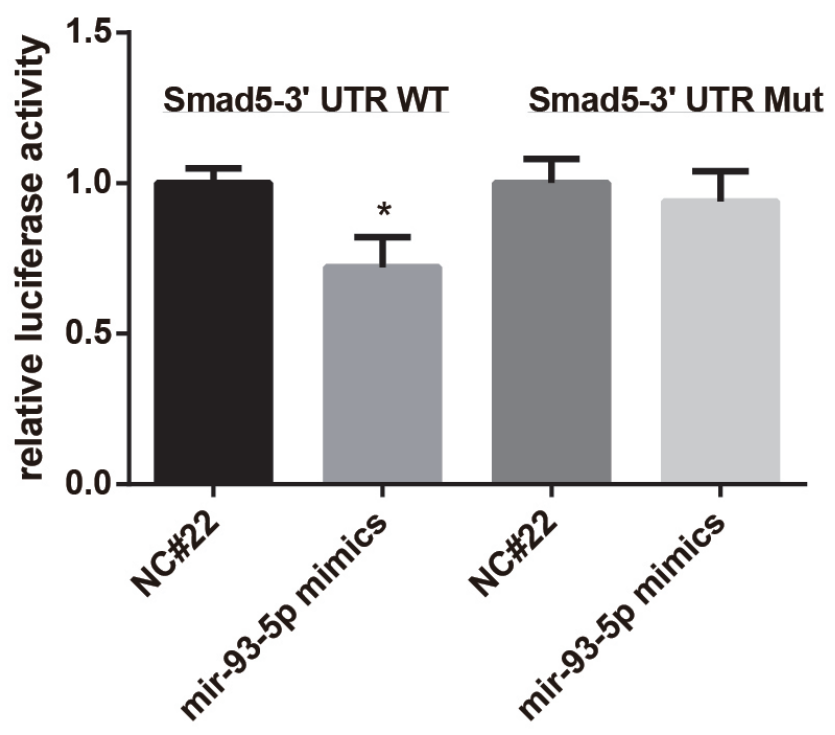

Figure 2: Dual luciferase reporter gene assay showed that Smad5 was a target of miR-93-5p. A. we synthesized a target sequence WT (GCACTTT) and then a mutant sequence Mut (CGTGAAA) through site-directed mutagenesis in WT; B. the luciferase activity was recorded and compared.

Note: NC\#22, miR-93-5p negative control; * $P<0.05$ in comparison with the group transfected with NC\#22 and pSmad5-WT. 
Table 2: The PMWTs of all groups at different timing after lentivirus transfection

\begin{tabular}{|c|c|c|c|}
\hline & $7 d$ & 9d & 14d \\
\hline L/anti-miR-93-5p group & $3.23 \pm 0.25^{*}$ & $3.35 \pm 0.31^{*}$ & $3.42 \pm 0.35^{*}$ \\
\hline Smad5 shRNA group & $6.11 \pm 0.54^{\#}$ & $6.24 \pm 0.70^{\#}$ & $6.30 \pm 0.75^{\#}$ \\
\hline L/anti-miR-93-5p + Smad5 shRNA group & $4.11 \pm 0.43^{\# *}$ & $4.24 \pm 0.46^{\# *}$ & $4.35 \pm 0.50^{\# *}$ \\
\hline Blank virus group & $4.09 \pm 0.38^{\#^{*}}$ & $4.18 \pm 0.49^{\# *}$ & $4.31 \pm 0.51^{\# *}$ \\
\hline PBS control group & $4.13 \pm 0.51^{\#^{*}}$ & $4.22 \pm 0.45^{\#^{*}}$ & $4.37 \pm 0.55^{\# *}$ \\
\hline
\end{tabular}

PMWT, paw mechanical withdrawal threshold; PBS, phosphate buffered saline; ${ }^{*}, P<0.05$ in comparison with the L/antimiR-93-5p group; \#, $P<0.05$ in comparison with the Smad5 shRNA group.

27]. In consideration of the proved biological function in bone metastases of mir-93 and Smad5, we hypothesized that miR-93/Smad4 may promote the progression of morphine tolerance in patients with BCP. Our result showed that miR-93 was upregulated while Smad5 was downregulated in the mice with BCP, suggesting that miR-93 was negatively correlated with Smad5. As previous studies suggested, up-regulation of miR-93 was detected in cervical carcinoma by Wang et al., in lung cancer by $\mathrm{Du}$ et al., and in head and neck squamous cell carcinoma by Li et al., indicating that miR-93 may contribute to the metastasis and invasion of cancers [28-30]. Also, Kang et al. reported that breast cancer bone metastasis was promoted through the Smad tumor suppressor pathway [31]. Along with statistical analysis, our dual luciferase reporter gene assay, verified that miR93 could directly suppress the expression of Smad5. With these findings, we go further to investigate the role of miR-93 and Smad5 in the formation of morphine tolerance in BCP mouse model.

As suggested, miR-93 and Smad5 closely correlated with cancer metastasis $[30,32]$. When Lewis lung cancer cell metastasize to bone, such algogenic substances as endothelins, prostaglandins, proteases, protons, bradykinin, and tyrosine kinase activators will be released by the cancer cells as well as their associated stromal cells, leading to induction of sensitization and activation of sensory nerves that innervate the bone [33, 34]. In this way, we believed that miR-93 suppressing Smad5 may contribute to the exacerbation of BCP. Using Western Blot assay, RT-qPCR and immunohistochemistry, we found that, in comparison with the healthy mice, the mice with BCP presented differential expression of miR93 and Smad5. In the bone metastasis, bone damage is not directly caused by cancer/stromal cells but by the receptor activator of nuclear factor $\kappa-\mathrm{B}$ ligand (RANKL) activated by them. The activation of RANKL enhances the binding activity between RANKL and RANK (its receptor), leading to expression of osteoclasts $[4,35]$. Aditi Gupta et al. reported that, the expression of RANKL could be knocked down by phosphorylation of Smad5 suppression, therefore reducing osteoclast differentiation and consequently lessening bone loss and BCP [36]. We also learned that activation of PI3K $\gamma / \mathrm{Akt}$ pathway, downstream of miR-93, could activate spinal microglia through MCP-1 and mediates BCP [37, 38]. Taken together, miR-93/Smad5 is suggested as an important mediator in BCP.

As observed, the mice in the morphine model had a significantly higher miR-93 expression and a lower Smad5 expression than the other groups. After we lentivirus transfection with L/anti-miR-93-5p and/ or Smad5 shRNA, we found that the PMWT changed with the expression of miR-93 and Smad5. Our results suggested that downregulation of miR-93 expression in the L/anti-miR-93-5p led to lower PMWTs while downregulation of Smad5 to elevated PMWTs. From this result, we think that miR-93/Smad5 interaction may participate in the formation of morphine tolerance. One possible explanation is that, as BCP progressed, the constant dose morphine fails to produce the analgesic effect as it did at the very start. In addition, Morphine realize it analgesic function trough targeting $\mu$ opioid receptor (MOR). Desensitization and trafficking of $\mu$ opioid receptor has been widely accepted as a main reason for morphine tolerance [39-41]. By regulating such transcription factors of OPRM (MOR gene) as NF$\kappa \mathrm{B}$ through different pathways, miR-93 and Smad5 may participate in the formation of morphine tolerance [36, 42]. In the present study, we only identify a relation of miR-93 and Smad5 to morphine tolerance while leave the mechanism how miR-93 and Smad5 influence morphine tolerance to future study. Above all, our study is a preliminary one. However, these results, if verified with more detailed studies, may be of great significance in the treatment of morphine tolerance. To sum up, we provide direct evidence that Smad5 is a target of miR93. By regulation Smad5 through miR-93, morphine tolerance may be controlled through regulating $\mathrm{BCP}$ or other causative factors. 


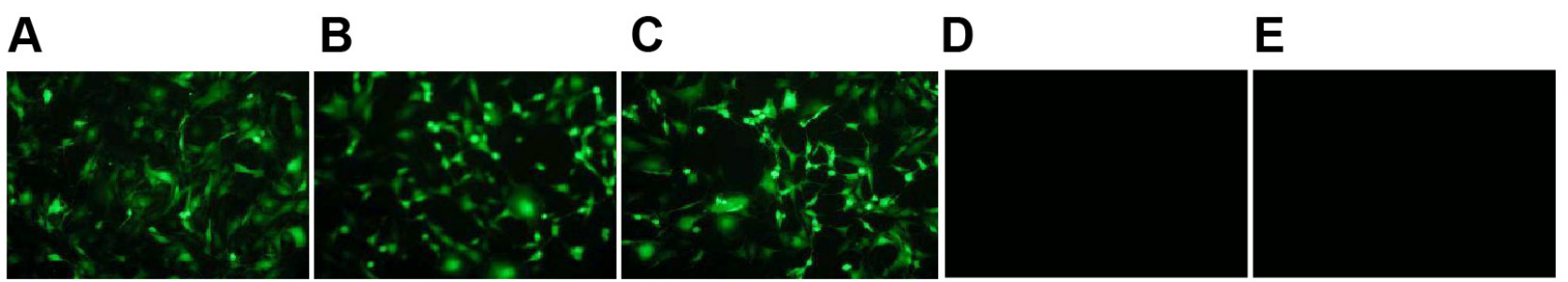

Figure 3: At 14 days after lentivirus transfection, L5 spinal marrows from all five groups were extracted and the transfection efficiency was evaluated. A. represents the L/anti-miR-93-5p group; B. represents the Smad5 shRNA group; C. represents the L/anti-miR-93-5p + Smad5 shRNA group; D. represents the blank virus group; E. represents the blank control group.

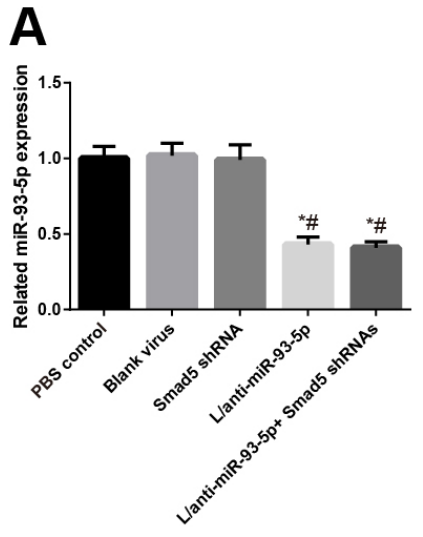

$\mathbf{E}$
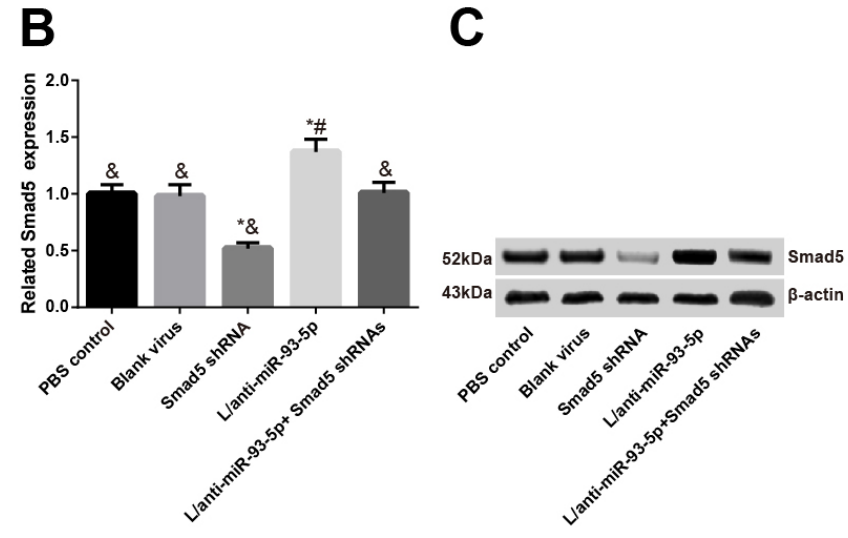

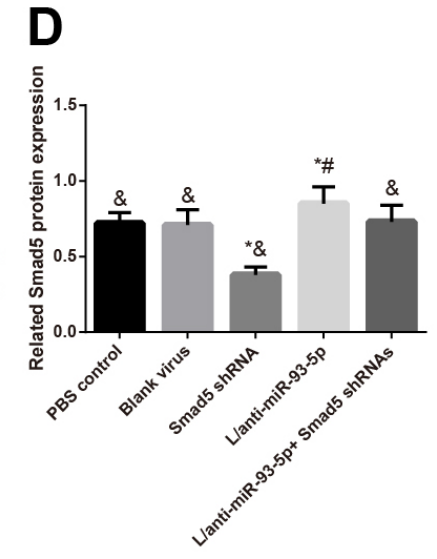

$\mathbf{F}$
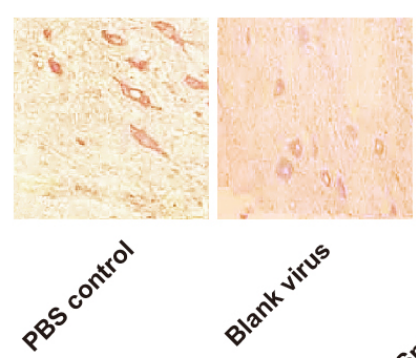

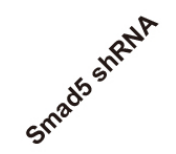

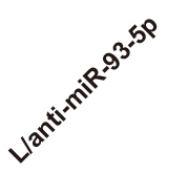

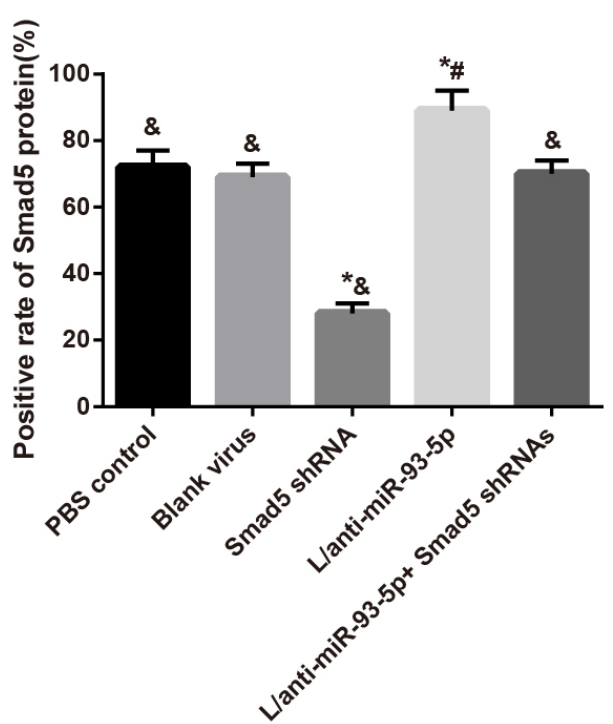

Figure 4: At 14 days after lentivirus transfection, L5 spinal marrows from all five groups were extracted and miR93-5p expression and Smad5 mRNA and protein expressions were detected. A. miR-93-5p expression; B. Smad5 mRNA expression; C. electrophoretogram of Smad5 protein by Western blot; D. alternations of Smad5 protein expression; E. Smad5 protein expression by immunohistochemistry; F. semi-quantitative Smad5 protein expression by immunohistochemistry.

Note: *, $P<0.05$ in comparison with the blank virus group; \#, $P<0.05$ in comparison with the Smad5 shRNA group; \&, $P<0.05$ in comparison with the L/anti-miR-93-5p group. 


\section{MATERIALS AND METHODS}

\section{Ethic statement}

This study was conducted following the recommendations proposed by International Association for the Study of Pain. The protocol was approved by the Animal Ethics Committee in Xiangya Hospital and Ningbo No.2 Hospital.

\section{Construction of L/anti-miR-93-5p lentivirus and Smad5 lentiviral shRNA vector}

The present study used the three-plasmid system; three packaging plasmids were pGLV-H1-GFP+Puro, PGP1-VSVO, PG-P2-REV [20]. The recombinant lentiviral vectors of anti-miR-93-5p and Smad5 shRNA was synthesized by Shanghai GenePharma Co., Ltd and verified through sequencing. The sequence of anti-miR-93-5p was 5'-GAUGGACGUGCUUGUCGUGAAAC-3' and that of Smad5 shRNA was 5'-AATTACTTTTATTTTGCATTTT-3'. In a 96-well plate, HEK $293 \mathrm{~T}$ cells were seeded $\left(0.5 \times 10^{4}\right.$ cells/well) and cultured with 10\%-fetal bovine serum (FBS) containing DMEM medium (100 $\mu \mathrm{L} /$ well; overnight $)$. On the next day, DMEM containing $10 \%$ FBS and polybrene (5 $\mu \mathrm{g} / \mathrm{ml}$ ) was used to dilute the lentiviruses to concentrations in gradient. After that, the culture solution was removed and replaced with $100 \mu \mathrm{L}$ lentivirus diluent. Three duplicated wells were set for wells at different dilution ratios of a lentivirus. A blank virus group and a phosphate buffered saline (PBS) control group were built. After overnight incubation, cells continued to be cultured with $100 \mu \mathrm{L}$ complete medium for 48 hours. Under a micro scope, the GFP-positive cells were counted in each transduction unit (TU) and the titer was calculated as: $\mathrm{TU} / \mathrm{ml}=[($ infected cells/field $) \times($ fields $/$ well $)] /$ Volume virus $(\mathrm{ml}) \times$ dilution factor.

\section{Animal modeling}

From the Nanjing Junke biological engineering co., LTD (Nanjing, China), male C57BL/6 mice ( $\mathrm{n}=90$; age range of $6 \sim 8$ weeks old; weight range of 18 23 g) were bought. Of 90 mice, 70 were randomly selected for building a BCP model by injecting Lewis lung cancer cells $\left(2 \times 10^{6}\right.$; Shanghai FengShou biological technology co., LTD) suspended with $10 \mu \mathrm{L}$ PBS into the bone cavity of each mouse [21]. The 50\% paw mechanical withdrawal threshold (PMWT) was recorded to decide on the validity of the model before modeling and at 3 days, 6 days and 9 days after modeling [22].

\section{Animal grouping and preparation of morphine tolerance model}

The mice with $\mathrm{BCP}(\mathrm{n}=70)$ were randomly allocated into saline model group $(\mathrm{n}=10)$ and morphine model group $(\mathrm{n}=60)$. The 20 healthy controls were contrastively divided into saline control group $(\mathrm{n}=$ 10) and morphine control group $(\mathrm{n}=10)$. After 6 days of successfully mouse modeling of BCP, the morphine control group and the morphine model group received hypodermic injection of morphine $(10 \mathrm{mg} / \mathrm{kg}$; twice a day) [23]. While the saline model group and the morphine model group had hypodermic injection of normal saline (NS) $(10 \mathrm{mg} / \mathrm{kg}$; twice a day). After 6 days of BCP mouse modeling, PMWT was determined at the $1^{\text {st }}, 3^{\text {rd }}, 5^{\text {th }}$ and $7^{\text {th }}$ after injection of morphine to confirm whether a morphine tolerance model was successfully built. In each group, 10 mice were killed at 7-day long injection for the detection of miR-93 and Smad5. The other 50 mice of the morphine model group continued to be kept alive until the next experiment.

\section{Transfection with lentivirus}

According to the transfection scheme, the 50 mice of the morphine model group were randomly assigned to L/ anti-miR-93 group, Smad5 shRNA group, L/anti-miR-93 + Smad5 shRNA group, blank group (lentivirus-free) and PBS control group. All the five groups were treated with venous injection of corresponding virus suspension or equal sterile PBS solution; all the virus suspensions were of same titer and same dosage. Following the construction of morphine model (at the $7^{\text {th }}$ after injection of morphine), PMWTs at 7, 9 and 14 days after injection were recorded.

\section{Measurement of PMWT}

The measurement of PMWT was conducted based on the method proposed in a previous study [24]. The mice to be tested were placed in a transparent perspex cage $(26 \mathrm{~cm}$ $\times 14 \mathrm{~cm} \times 26 \mathrm{~cm}$ ) and the cage was put on a 22-cm high net rack (each grid of $0.5 \mathrm{~cm} \times 0.5 \mathrm{~cm}$ ). About $15 \sim 20$ minutes later, vonFrey cilium (Stoeling, America) of different stresses $(0.18,0.25,0.6,1.3,3.8,5.4,7.6$ and $9.7 \mathrm{~g})$ was used to poke the mice right at the sensitive postmedian of their paws. A cycle of stimulation at a force consisted of 5 pokes (6 8 seconds/poke). Each poke was conducted at $\geq 2$ minutes after the last poke when the mice regain its calmness. The least stress causing $\geq 2$ paw withdrawals was described as PMWT.

\section{Luciferase reporter gene assay}

Using PicTar, TagetScan, miRanda and miRDB, the binding site between microR-93-5p and Smad5 was predicted, finding that 776-782 nt (GCACTTT) at Smad5 mRNA3'-UTR region was the one in search. Based on this finding, we synthesized a target sequence WT (GCACTTT) and then a mutant sequence Mut (CGTGAAA) through site-directed mutagenesis in WT. After receiving digestion with Xho I and Not I restriction enzyme, pmiR-RB-REPORTTM plasmids combined 
with synthesized WT or Mut to construct recombinant plasmids, pSmad5-WT or pSmad5-Mut. The recombinant plasmids were validated through sequencing. HEK 293T cells were used for cell transfection and luciferase assay. Receiving different transfection schemes, cells were allocated into four groups: Group A, transfected with NC\#22 (miR-93-5p negative control) and pSmad5WT; Group B, transfected with miR-93-5p mimics and pSmad5-WT; Group C, transfected with NC\#22 and pSmad5-Mut; Group D, transfected with miR-93-5p mimics and pSmad5-Mut. After transfection, cells were seeded into culture medium-containing $(100 \mu \mathrm{L} /$ well $)$ 96-well plates at a cell concentration of $1.5 \times 10^{4}$ cells/ well, followed by incubation in an incubator of saturated humidity $\left(37^{\circ} \mathrm{C}, 5 \% \mathrm{CO}_{2}, 24\right.$ hours $)$. On the next day, the culture medium was renewed $(50 \mu \mathrm{L} /$ well $)$. Meanwhile, $10 \mu \mathrm{L}$ reduced serum medium OPTI-MEM (Thermo Fisher Scientific, America) diluting miR-93-5p or NC\#22 (100 nmol/L), $15 \mu \mathrm{L}$ OPTI-MEM diluting pSmad5-WT or pSmad5-Mut (100 ng) and $25 \mu \mathrm{L}$ OPTI-MEM diluting Lipofectamine $2000(0.25 \mu \mathrm{L})$ were intensively mixed together after 5-minute reaction. After 20-minute long incubation at room temperature, the mixture $(50 \mu \mathrm{L})$ was put into a well for cell incubation. Together with the 50 $\mu \mathrm{L}$ medium, each well contained culture solution of 100 $\mu \mathrm{L}$. For each group of cells, 3 duplicated well were set. At 6 hours after transfection, the culture solution was renewed again with $100 \mu \mathrm{L}$ culture medium. At 48 hours after transfection, dual luciferase reporter gene assays were conducted with luciferase reporter gene assay kit (Beyotime Biotechnology, China) and comparisons were made among the four groups in their luciferase activity (hRluc/hLuc).

\section{Specimen preparation and immumohistochemical staining}

After receiving deep anesthesia with Nembutal, mice in the four groups were treated with thoracotomy; a cannula was intubated to the ascending aorta, followed by swill with $0.9 \%$ saline $(20 \mathrm{~mL})$ to remove the blood in a short time and then perfusion (20 minutes) for fixation with $4 \%$ paraformaldehyde-containing phosphate buffer $(0.1 \mathrm{~mol} / \mathrm{L}, \mathrm{pH}=7.4)$. Following the perfusion, L5 spinal marrow was extracted and then fixed $\left(4^{\circ} \mathrm{C}\right.$, $4 \sim 6$ hours). The fixed L5 spinal marrow then was immersed first in $20 \%$ and then $30 \%$ sucrose solution till it sank to the bottom. After that, the spinal marrow was frozen and then made into serial sections $(40-\mu \mathrm{m})$. The immumohistochemical staining was conducted with avidin biotin complex (ABC) system. The sections were blocked with 5\% goat serum and incubated in rabbit-anti-Smad5 (1:800; ab92698; Abcam, America) first at $37^{\circ} \mathrm{C}$ for 2 hours and then cultured at $4^{\circ} \mathrm{C}$ for 48 hours. On the next day, the sections were rewarmed, followed by incubation with Biotin labeled goat-anti-rabbit $\operatorname{IgG}\left(37^{\circ} \mathrm{C}, 2\right.$ hours $)$ and then with $\mathrm{ABC}$ complex $\left(37^{\circ} \mathrm{C}, 1\right.$ hours $)$. During the immumohistochemical staining, all the antibodies were diluted with $0.01 \mathrm{~mol} / \mathrm{L}$ PBS ( $\mathrm{pH} 7.4)$ and the sections were rinsed thoroughly with PBS before they moving on to the next step. After staining, the sections received DAB (short for diaminobenzidine coloration, mounting, drying, dehydration, hyalinization and blockage by gelatin. For the sections of the negative control group, the primary antibody was instituted with PBS. Under OLYMPUS IX81 light microscope (Olympus, Tokyo, Japan; × 200), 10 fields were randomly selected to observe the sections; cells in dark brown were considered as positive. The percentage of positive cells was recorded on a scale of 0 to 3 , with 0 indicating less than $10 \%, 1$ representing $10 \%$ to $25 \%, 2$ representing $25 \%$ to $50 \%, 3$ representing more than $50 \%$. Then, we defined the above results as 0 score (negative), 1 2 scores (weak positive), 3 4 scores (positive) and $5 \sim$ scores (strong positive). Finally, 0 score was defined as negative expression and 1 5 scores were defined as positive expression. The positive intensity was analyzed with Image-Pro Plus 6.0 software (Media Cybernetics, Silver Spring, MD, USA).

\section{Real-time fluorescence quantitative polymerase chain reaction (RT-qPCR)}

Through the same method described above, L5 spinal marrow was collected with Diethy pyrocarbonate (DEPC)-containing centrifugal tubes $(2 \mathrm{~mL})$, followed by RNA extraction with Trizol (TAKARA, Japan) and measurement of RNA concentration and RNA purification with an $\mu$ Ltraviolet spectrophotometer (Eppendorf, German). A RNA sample of $1000 \mathrm{ng}$ was used for reverse transcription (RT) according to the instruction of PrimeScriptTM RT Regeant Kit (TAKARA, Japan). The reverse transcription system included $5 \times$ PrimeScriptTM Buffer (4 $\mu \mathrm{L})$, PrimeScriptTM RT Enzyme Mix I (1 $\mu \mathrm{L})$, $50 \mu \mathrm{M}$ OligodT Primer $(1 \mu \mathrm{L}), 100 \mu \mathrm{M}$ Random 6 mers $(1 \mu \mathrm{L})$ and RNase Free dH2O $(13 \mu \mathrm{L})$. The RT protocol was one cycle at $37^{\circ} \mathrm{C}$ for 30 minutes and then one cycle at $85^{\circ} \mathrm{C}$ for 6 seconds. The RT-qPCR was conducted with SYBR Premix Ex Taq II kit (TAKARA, Japan). The RTqPCR system included RT product $(1 \mu \mathrm{L})$, SYBR Green I Premix Ex Taq II $(2 \times)(10 \mu \mathrm{L}), 10 \mathrm{uM}$ PCR Forward Primer $(0.8 \mu \mathrm{L}), 10 \mathrm{uM}$ PCR Reverse Primer $(0.8 \mu \mathrm{L})$, ROX Reference Dye $(50 \times)(0.4 \mu \mathrm{L})$ and $\mathrm{dH} 2 \mathrm{O}(7 \mu \mathrm{L})$. The RT-qPCR protocol was one cycle of 30 seconds at $95^{\circ} \mathrm{C}$, and then 40 cycles of 5 seconds at $95^{\circ} \mathrm{C}, 30$ seconds at $56^{\circ} \mathrm{C}$, and 30 seconds at $72^{\circ} \mathrm{C}$. The calculation of relative expression of target gene was conducted with $2^{-\Delta \Delta C T}$ method; the internal reference for miR-93-5p was snRNA U6 and the internal reference for Smad5 was GAPDH. The primer sequences were listed in Table 3.

\section{Western blot assay}

The L5 spinal marrow was collected with a DEPC centrifugal tube as described above. After that, $100 \mu \mathrm{L}$ 
Table 3: List of primer sequences for RT-qPCR

\begin{tabular}{lcc}
\hline Gene & Forward primer & Reverse primer \\
\hline MiR-93-5p & 5'-AGTCTCTGGCTGACTACATCACAG-3' & 5'-CTACTCACAAAACAGGAGTGGAATC-3' \\
U6 & 5'-GCTTCGGCAGCACATATACTAAAAT-3' & 5'-CGCTTCACGAATTTGCGTGTCAT-3' \\
Smad5 & 5'-CTTGGATGGACGTCTGCAAG-3' & 5'-CATGGTGAAAGTTGCAGTTC-3' \\
GAPDH & 5'-GCTTGAAGGTGTTGCCCTCAG-3' & 5'-AGAAGCCAGCGTTCACCAGAC-3' \\
\hline
\end{tabular}

RT-qPCR, real-time fluorescence quantitative polymerase chain reaction.

RIPA lysis buffer (ShineGene Molecular Biotechnology, China) was added to decompose the marrow, followed by homogenate by intensive vibration on ice. At 30 minutes after homogenate, protein extraction was conducted in strict accordance with the instructions. Subsequently, protein measurement was conducted with bicinchoninic acid (BCA) protein quantization kit (Beyotime Biotechnology, China). On basis of the protein concentration, the volume of the upper sample was calculated and the upper sample was mixed with loading buffer at a ratio of $1: 1$, followed by 5-minute long hot bath and then sample adding. Thus, a sodium dodecyl sulfate- polyacrylamide gel electrophoresis (SDSPAGE) weldwood was prepared for electrophoresis. The electrophoresis was performed with 5\% spacer gel and 12\% separation gel first at $80 \mathrm{~V}$ and then 100 V. Subsequently, the gel were incised, chamfered and sandwiched, followed by electric transfection $\left(4^{\circ} \mathrm{C}\right.$, $200 \mathrm{~mA}, 2$ hours) to a membrane. Next, the membrane received blockage with 5\% skim milk (room temperature, 1 hour) and tert-butyldimethylsilyl-Tween (TBST) rinse (10 minutes $\times 3$ times), followed by incubation $\left(4^{\circ} \mathrm{C}\right.$, overnight) with monoclonal rabbit-anti-Smad5 antibody (1:1000; ab92698, Abcam, America) or polyclonal rabbit-anti- $\beta$-actin antibody $(1: 4000$; ab129348, Abcam, America). On the next day, the membrane was again rinsed (10 minutes $\times 3$ times) and continued to be incubated with HRP-labeled goat-anti-rabbit IgG at room temperature for 1 hour (Sigma, America). After that, the membrane was rinsed for coloration with an ECL kit and scanned with a gel imaging system. For analysis on the target bands, ImageJ software was used. In the calculation of Smad5 expression, $\beta$-actin was the internal reference.

\section{Statistical analyses}

For data analyses, SPSS21.0 was used (SPSS Inc., Chicago, IL, USA). Measurement date was expressed as mean \pm standard deviation. Among-group comparisons were conducted by variance analysis and between-group comparisons were by $\mathrm{t}$ test. Pearson analysis was used for correlation analysis. All the tests were two-tailed; $P<0.05$ indicated significant difference.

\section{ACKNOWLEDGMENTS}

This study was supported by the National Natural Science Foundation of China (No. 81402224, No. 81401838), the Provincial Science Foundation of Hunan (No. 2015JJ3139), the Development and Reform Commission of Hunan Province ([2014]658-8), the Science and Technology Bureau of Hunan Province (2012FJ6001), the Science and Technology Office of Changsha City (K1203040-31), the Health and Family Planning Commission of Hunan Province (B2014-12), the Administration of Traditional Chinese Medicine of Hunan Province (No. 2015116) and the Open-End Fund for the Valuable and Precision Instruments of Central South University (CSUZC201639). We would like to acknowledge the reviewers for their helpful comments on this paper.

\section{CONFLICTS OF INTEREST}

The authors declare no conflicts of interest.

\section{REFERENCES}

1. Pockett RD, Castellano D, McEwan P, Oglesby A, Barber BL, Chung K. The hospital burden of disease associated with bone metastases and skeletal-related events in patients with breast cancer, lung cancer, or prostate cancer in Spain. European journal of cancer care. 2010; 19:755-760.

2. Jimenez-Andrade JM, Mantyh WG, Bloom AP, Ferng AS, Geffre CP, Mantyh PW. Bone cancer pain. Annals of the New York Academy of Sciences. 2010; 1198:173-181.

3. Kim T, Jung Cha H, Won Kim J, Seong J, Jae Lee I. High dose, compartmental target volume may improve patient outcome after radiotherapy for pelvic bone metastases from hepatocellular carcinoma. Oncotarget. 2016. doi: 10.18632/ oncotarget.9767.

4. Mantyh PW. Bone cancer pain: from mechanism to therapy. Current opinion in supportive and palliative care. 2014; 8:83-90.

5. Honore P, Luger NM, Sabino MA, Schwei MJ, Rogers SD, Mach DB, O'Keefe PF, Ramnaraine ML, Clohisy DR, Mantyh PW. Osteoprotegerin blocks bone cancer-induced 
skeletal destruction, skeletal pain and pain-related neurochemical reorganization of the spinal cord. Nat Med. 2000; 6:521-528.

6. Niu DG, Peng F, Zhang W, Guan Z, Zhao HD, Li JL, Wang KL, Li TT, Zhang Y, Zheng FM, Xu F, Han QN, Gao P, et al. Morphine promotes cancer stem cell properties, contributing to chemoresistance in breast cancer. Oncotarget. 2015; 6:3963-3976. doi: 10.18632/oncotarget.2894.

7. Portenoy RK. Treatment of cancer pain. Lancet. 2011; 377:2236-2247.

8. Cao F, Gao F, Xu AJ, Chen ZJ, Chen SS, Yang H, Yu HH, Mei W, Liu XJ, Xiao XP, Yang SB, Tian XB, Wang XR, Tian YK. Regulation of spinal neuroimmune responses by prolonged morphine treatment in a rat model of cancer induced bone pain. Brain research. 2010; 1326:162-173.

9. Yang IP, Tsai HL, Hou MF, Chen KC, Tsai PC, Huang SW, Chou WW, Wang JY, Juo SH. MicroRNA-93 inhibits tumor growth and early relapse of human colorectal cancer by affecting genes involved in the cell cycle. Carcinogenesis. 2012; 33:1522-1530.

10. Liu S, Patel SH, Ginestier C, Ibarra I, Martin-Trevino R, Bai S, McDermott SP, Shang L, Ke J, Ou SJ, Heath A, Zhang KJ, Korkaya H, Clouthier SG, Charafe-Jauffret E, Birnbaum D, et al. MicroRNA93 regulates proliferation and differentiation of normal and malignant breast stem cells. PLoS genetics. 2012; 8:e1002751.

11. Long J, Wang Y, Wang W, Chang BH, Danesh FR. Identification of microRNA-93 as a novel regulator of vascular endothelial growth factor in hyperglycemic conditions. The Journal of biological chemistry. 2010; 285:23457-23465.

12. Tang Q, Zou Z, Zou C, Zhang Q, Huang R, Guan X, Li Q, Han Z, Wang D, Wei H, Gao X, Wang X. MicroRNA-93 suppress colorectal cancer development via Wnt/betacatenin pathway downregulating. Tumour Biol. 2015; 36:1701-1710.

13. Ohta K, Hoshino H, Wang J, Ono S, Iida Y, Hata K, Huang SK, Colquhoun S, Hoon DS. Microrna-93 activates c-met/ pi3k/akt pathway activity in hepatocellular carcinoma by directly inhibiting pten and cdkn1a. Oncotarget. 2015; 6:3211-3224. doi: 10.18632/oncotarget.3085.

14. Fang L, Du WW, Yang W, Rutnam ZJ, Peng C, Li H, O'Malley YQ, Askeland RW, Sugg S, Liu M, Mehta T, Deng Z, Yang BB. MiR-93 enhances angiogenesis and metastasis by targeting LATS2. Cell cycle. 2012; 11:4352-4365.

15. Qu MH, Han C, Srivastava AK, Cui T, Zou N, Gao ZQ, Wang QE. miR-93 promotes TGF-beta-induced epithelialto-mesenchymal transition through downregulation of NEDD4L in lung cancer cells. Tumour Biol. 2015.

16. Xiao F, Qiu H, Cui H, Ni X, Li J, Liao W, Lu L, Ding K. MicroRNA-885-3p inhibits the growth of HT-29 colon cancer cell xenografts by disrupting angiogenesis via targeting BMPR1A and blocking BMP/Smad/Id1 signaling. Oncogene. 2015; 34:1968-1978.
17. Marks-Bluth J, Khanna A, Chandrakanthan V, Thoms J, Bee T, Eich C, Kang YC, Knezevic K, Qiao Q, Fitch S, Oxburgh L, Ottersbach K, Dzierzak E, de Bruijn MF, Pimanda JE. SMAD1 and SMAD5 Expression Is Coordinately Regulated by FLI1 and GATA2 during Endothelial Development. Molecular and cellular biology. 2015; 35:2165-2172.

18. Lulli V, Buccarelli M, Martini M, Signore M, Biffoni M, Giannetti S, Morgante L, Marziali G, Ilari R, Pagliuca A, Larocca LM, De Maria R, Pallini R, et al. Mir-135b suppresses tumorigenesis in glioblastoma stem-like cells impairing proliferation, migration and self-renewal. Oncotarget. 2015; 6:37241-37256. doi: 10.18632/ oncotarget.5925.

19. Tasca A, Stemig M, Broege A, Huang B, Davydova J, Zwijsen A, Umans L, Jensen ED, Gopalakrishnan R, Mansky KC. Smad1/5 and Smad4 expression are important for osteoclast differentiation. Journal of cellular biochemistry. 2015; 116:1350-1360.

20. Buchschacher GL, Jr., Wong-Staal F. Development of lentiviral vectors for gene therapy for human diseases. Blood. 2000; 95:2499-2504.

21. Zhu M, Shen Y, Wu X, Zhang Z. [overexpression of heme oxygenase 1 in spinal cord alleviates bone cancer pain in mice]. Zhonghua Yi Xue Za Zhi. 2015; 95:1341-1344.

22. Wang D, Ren BX, Liu CL, Zhu J, Zhang J, Zhang W, Mei FM, Gu XP, Ma ZL. [role of cannabinoid 2 receptor in the development of bone cancer pain]. Zhonghua Yi Xue Za Zhi. 2012; 92:440-443.

23. Yan X, Huang X, Huang D. [nmda receptor and nos in morphine tolerance in mice with bone cancer]. Zhong Nan Da Xue Xue Bao Yi Xue Ban. 2010; 35:458-463.

24. Kim SH, Chung JM. An experimental model for peripheral neuropathy produced by segmental spinal nerve ligation in the rat. Pain. 1992; 50:355-363.

25. Ghelardini C, Di Cesare Mannelli L, Bianchi E. The pharmacological basis of opioids. Clin Cases Miner Bone Metab. 2015; 12:219-221.

26. Williams JT, Christie MJ, Manzoni O. Cellular and synaptic adaptations mediating opioid dependence. Physiological reviews. 2001; 81:299-343.

27. Bohn LM, Gainetdinov RR, Lin FT, Lefkowitz RJ, Caron MG. Mu-opioid receptor desensitization by beta-arrestin-2 determines morphine tolerance but not dependence. Nature. 2000; 408:720-723.

28. Wang L, Wang Q, Li HL, Han LY. Expression of mir200a, mir93, metastasis-related gene reck and mmp2/mmp9 in human cervical carcinoma-relationship with prognosis. Asian Pac J Cancer Prev. 2013; 14:2113-2118.

29. Du L, Zhao Z, Ma X, Hsiao TH, Chen Y, Young E, Suraokar M, Wistuba I, Minna JD, Pertsemlidis A. Mir-93-directed downregulation of dab2 defines a novel oncogenic pathway in lung cancer. Oncogene. 2014; 33:4307-4315.

30. Li G, Ren S, Su Z, Liu C, Deng T, Huang D, Tian Y, Qiu Y, Liu Y. Increased expression of mir-93 is associated with 
poor prognosis in head and neck squamous cell carcinoma. Tumour Biol. 2015; 36:3949-3956.

31. Kang Y, He W, Tulley S, Gupta GP, Serganova I, Chen CR, Manova-Todorova K, Blasberg R, Gerald WL, Massague J. Breast cancer bone metastasis mediated by the smad tumor suppressor pathway. Proc Natl Acad Sci U S A. 2005; 102:13909-13914.

32. Liu IM, Schilling SH, Knouse KA, Choy L, Derynck R, Wang XF. TGFbeta-stimulated Smad1/5 phosphorylation requires the ALK5 L45 loop and mediates the promigratory TGFbeta switch. The EMBO journal. 2009; 28:88-98.

33. Mantyh P. Bone cancer pain: causes, consequences, and therapeutic opportunities. Pain. 2013; 154:S54-62.

34. Cai X, Luo J, Yang X, Deng H, Zhang J, Li S, Wei H, Yang C, Xu L, Jin R, Li Z, Zhou W, Ding J, et al. In vivo selection for spine-derived highly metastatic lung cancer cells is associated with increased migration, inflammation and decreased adhesion. Oncotarget. 2015; 6:22905-22917. doi: 10.18632/oncotarget.4416.

35. Pitari MR, Rossi M, Amodio N, Botta C, Morelli E, Federico C, Gulla A, Caracciolo D, Di Martino MT, Arbitrio M, Giordano A, Tagliaferri P, Tassone P. Inhibition of mir-21 restores rankl/opg ratio in multiple myelomaderived bone marrow stromal cells and impairs the resorbing activity of mature osteoclasts. Oncotarget. 2015; 6:27343-27358. doi: 10.18632/oncotarget.4398.

36. Gupta A, Cao W, Chellaiah MA. Integrin alphavbeta 3 and CD44 pathways in metastatic prostate cancer cells support osteoclastogenesis via a Runx2/Smad 5/receptor activator of NF-kappaB ligand signaling axis. Molecular cancer. 2012; 11:66.

37. Jin D, Yang JP, Hu JH, Wang LN, Zuo JL. MCP-1 stimulates spinal microglia via PI3K/Akt pathway in bone cancer pain. Brain research. 2015; 1599:158-167.

38. Guan X, Fu Q, Xiong B, Song Z, Shu B, Bu H, Xu B, Manyande A, Cao F, Tian Y. Activation of PI3Kgamma/ Akt pathway mediates bone cancer pain in rats. Journal of neurochemistry. 2015; 134:590-600.

39. Bailey CP, Connor M. Opioids: cellular mechanisms of tolerance and physical dependence. Current opinion in pharmacology. 2005; 5:60-68.

40. Martini L, Whistler JL. The role of mu opioid receptor desensitization and endocytosis in morphine tolerance and dependence. Current opinion in neurobiology. 2007; 17:556-564.

41. Lopez-Gimenez JF, Milligan G. Opioid regulation of mu receptor internalisation: relevance to the development of tolerance and dependence. CNS \& neurological disorders drug targets. 2010; 9:616-626.

42. Saba E, Jeon BR, Jeong DH, Lee K, Goo YK, Kwak D, Kim S, Roh SS, Kim SD, Nah SY, Rhee MH. A Novel Korean Red Ginseng Compound Gintonin Inhibited Inflammation by MAPK and NF-kappaB Pathways and Recovered the Levels of mir-34a and mir-93 in RAW 264.7 Cells. Evid Based Complement Alternat Med. 2015; 2015:624132. 